\title{
Yoga and pilates in the management of low back pain
}

\author{
Susan Sorosky $\cdot$ Sonja Stilp $\cdot$ Venu Akuthota
}

Published online: 6 November 2007

(C) Humana Press 2007

\begin{abstract}
Many interventions for the management of low back pain exist, however most have modest efficacy at best, and there are few with clearly demonstrated benefits once pain becomes chronic. Therapeutic exercise, on the other hand, does appear to have significant benefits for managing patients with chronic low back pain (CLBP) in terms of decreasing pain and improving function. In addition, because chronic pain is complex and does not fit a simple model, there have also been numerous trials investigating and demonstrating the efficacy of multidisciplinary pain programs for CLBP. It follows that interventions that treat more than one aspect of LBP would have significant benefits for this patient population. Yoga and Pilates which have, both been gaining in popularity over the last decade are two mind-body exercise interventions that address both the physical and mental aspects of pain with core strengthening, flexibility, and relaxation. There has been a slow evolution of these nontraditional exercise regimens into treatment paradigms for LBP, although few studies
\end{abstract}

S. Sorosky $(\bowtie)$

Desert Pain Institute, Phoenix, AZ, USA

e-mail: ssorosky@cox.net

S. Sorosky

Midwestern University, Glendale, AZ, USA

S. Stilp

Boulder Orthopedics, Boulder, CO, USA

S. Stilp · V. Akuthota

Department of Physical Medicine and Rehabilitation, University of Colorado School of Medicine, New York, USA

V. Akuthota

The Spine Center at the University of Colorado Hospital, Aurora, CO, USA examining their effects have been published. The following article will focus on the scientific and theoretical basis of using yoga and Pilates in the management of CLBP.

Keywords Low back pain - Yoga - Pilates . Therapeutic exercise $\cdot$ Core strengthening $\cdot$ Rehabilitation

Many interventions for the management of low back pain exist, however most have modest efficacy at best, and there are few with clearly demonstrated benefits once pain becomes chronic. Therapeutic exercise, on the other hand, does appear to have significant benefits for managing patients with chronic low back pain (CLBP). In a systematic review of the literature evaluating exercise therapy for non-specific CLBP, the authors concluded therapeutic exercise is effective in decreasing pain and improving function in this patient population [1]. Studies examining specific exercise techniques for LBP including core strengthening have reported even more promising results. In addition, because chronic pain presents with many facets and does not fit a simple model, there have also been numerous trials investigating and demonstrating the efficacy of multidisciplinary pain programs for CLBP. It follows that interventions that treat more than one aspect of LBP would have significant benefits for this patient population. Yoga and Pilates which have both been gaining in popularity over the last decade are two mind-body exercise interventions that address both the physical and mental aspects of pain with core strengthening, flexibility and relaxation. There has been a slow evolution of these nontraditional exercise regimens into treatment paradigms for LBP, although few studies examining their effects have been published. The following article will focus on the 
scientific and theoretical basis of using yoga and Pilates in the management of CLBP.

\section{Yoga}

Yoga is a form of mind-body exercise which couples physical exercise with mental focus through breathing and meditation. There has been a dramatic increase in the popularity of yoga in America over the last decade. In 1998, a national survey on the prevalence of adult yoga use in the United States estimated that 15.0 million American adults had used yoga at least once in their lifetime and 7.4 million during the previous year [2]. Participators reported using yoga for both wellness and health issues; specifically, $21 \%$ of respondents using yoga in the previous 12 months practiced yoga for back or neck pain. More recently, according to the Yoga Research and Education Council Report on Yoga statistics, there were 15 million Americans practicing yoga more than three times weekly in 2003 [3]. Today, yoga classes are a regular fixture at most health and fitness clubs; in addition, private yoga studios abound, offering instruction in different styles of yoga.

\section{Yoga background and styles}

Hatha yoga, the yoga typically practiced in the United States, is only one part of the non-sectarian philosophical system of yoga that emerged from the Indian culture approximately 4,000 years ago. Hatha yoga is comprised of three aspects which are integrated with one another including (1) asanas or physical postures, (2) pranayama or breathing exercises and (3) meditation or relaxation. The postures are designed to increase flexibility and strengthen the body in a controlled fashion as well as improve balance. These poses are performed standing, sitting, reclining, or inverted and may involve forward bends, backward bends, twists, or balancing (see Figs. 1 and 2). Breathing exercises link the postures together and help the mind focus. A Hatha yoga class concludes with a short meditation session during which the participant lies supine with eyes closed while shifting attention inwardly to create a relaxed state of being. A yoga instructor typically leads a group of practitioners through a class lasting 60-90 min.

There are many styles of Hatha yoga including Iyengar yoga, Viniyoga, Ashtanga yoga (also known as "power yoga"), and Bikram ("hot") yoga. Each of these forms of Hatha yoga has an unique approach. For example, Iyengar yoga is known for its emphasis on precise postural alignment and utilizes "props" including blocks and straps to assist in positioning. Iyengar yoga is the most commonly practiced style of yoga in the United States [3]. The

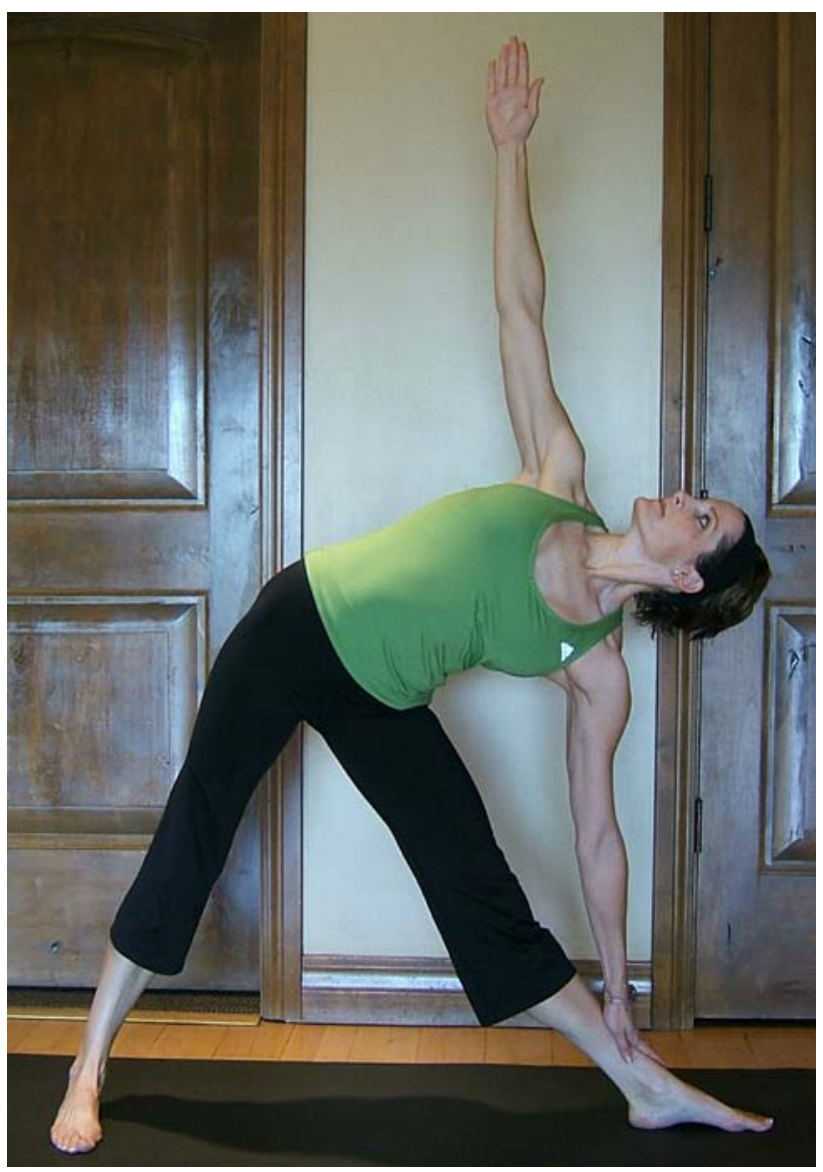

Fig. 1 Yoga pose-Triangle

Viniyoga style is a relatively easy style of yoga to learn and emphasizes the individualized nature of the yoga practice. Asthanga yoga is known for its vigorous flow of standardized postures linked together by breath. In our opinion, this style of yoga is typically more suited for the intermediate or advanced practitioner. Finally, in Bikram yoga, a series of postures, each held for a specific length of time are typically performed in intense heat $\left(105^{\circ} \mathrm{F}\right)$. It is notable that such temperatures may exacerbate heat-sensitive medical conditions.

\section{The yoga literature}

Literature evaluating the effectiveness of yoga for LBP is scant; a review of this subject revealed only four published randomized controlled studies. In 2004, Jacobs et al. conducted a feasibility study exploring an Iyengar yoga intervention for CLBP [3]. Fifty-two subjects who had made at least three visits to a health care provider for nonspecific LBP in the past 12 months were enrolled in this study. Participants were then randomly assigned to one of the two groups including 28 to the yoga group and 24 to 


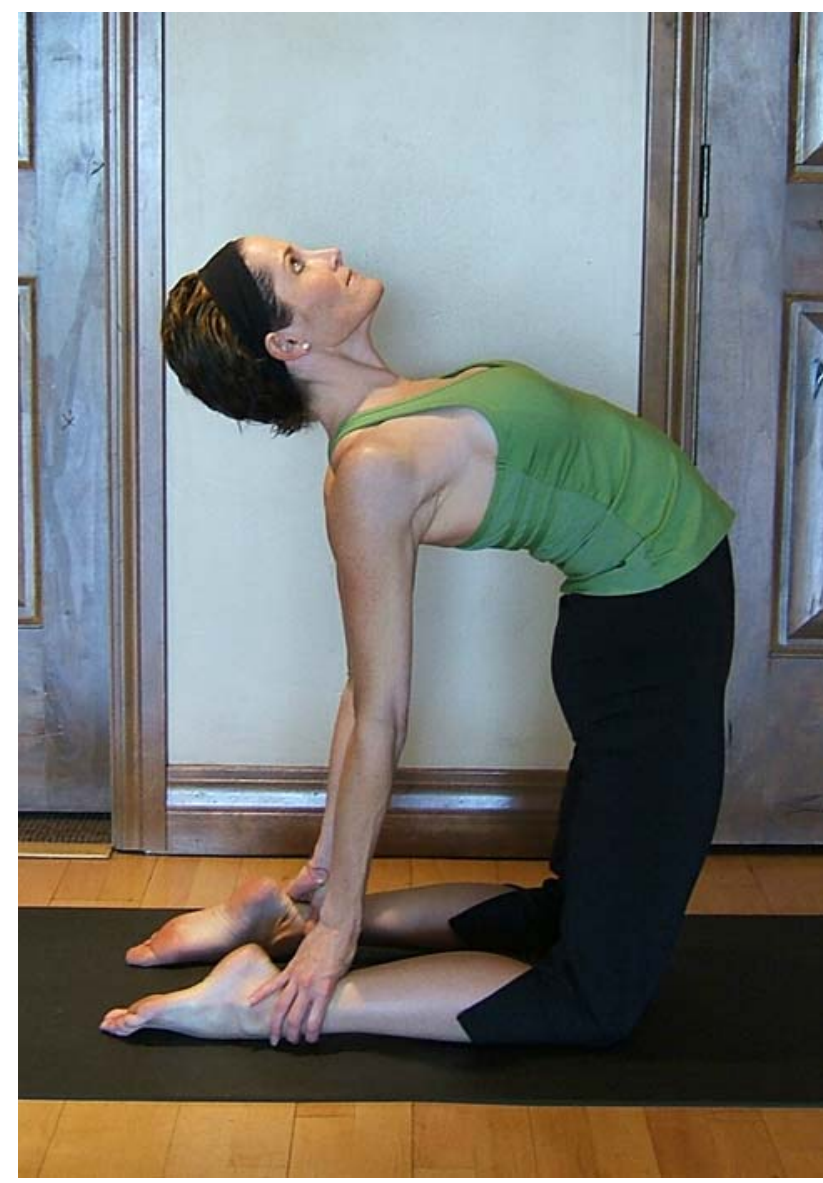

Fig. 2 Yoga pose-Camel

the wait-list control group who received a back pain educational booklet and then the same intervention 3 months later. The yoga protocol was based on the consensus opinion of eight senior Iyengar yoga instructors. It involved a semi-structured format with a predefined set of 28 postures from which the teacher could select individual poses in varying sequences based upon the needs of the class. Four experienced Iyengar instructors taught 90-min classes twice weekly for 12 weeks. The yoga participants were also encouraged to practice yoga at home for $30 \mathrm{~min}$, 5 days a week. The authors proposed primary and secondary outcome measures that would be assessed at baseline and 1, 3, and 6 month follow-ups, however only presented baseline characteristics of the two groups as well as adherence rates to the yoga intervention. Demographic and clinical baseline data of the two groups was similar with participants aged 18 to 65 (mean age 43.4) with an average visual analogue scale (VAS) of 4.2 and an average pain duration of 11.3 years. The authors also reported adherence with $64 \%$ of participants in the yoga group attending classes throughout the 3 month intervention period; on average, participants attended $15(66 \%)$ classes. They concluded, this study demonstrated the feasibility of developing a yoga protocol based on consensus by an expert panel of senior yoga teachers with moderately good adherence.

Galantino et al. also published a randomized controlled pilot study in 2004 studying the effects of a modified yoga protocol on patients with CLBP [4]. Twenty-two participants with nonspecific CLBP were enrolled in this study. Subjects were required to have had LBP greater than 6 months and a history of receiving two or more conservative medical interventions without prolonged relief. Participants were then randomly assigned to one of the two groups including 11 to the yoga group and 11 to the control group who received the same intervention after the study phase was completed. The yoga protocol was established by an "expert panel" of two yoga instructors with greater than 10 years experience and a physical therapist specializing in spine treatment and consisted of postures adapted for each individual. A single certified yoga instructor administered the 1 hour protocol twice weekly for 6 weeks. In addition, yoga participants were asked to practice yoga at home for $1 \mathrm{~h}$ per day as frequently as possible throughout the week.

Outcome measures including the Oswestry Disability Index (ODI), Beck Depression Inventory (BDI), and two secondary functional measurements of flexibility and balance (the Sit and Reach (SR) and Functional Reach (FR) tests) were assessed at baseline and at 6 weeks in both groups. In addition, yoga participants were given a series of questions for qualitative data analysis after each class and at 3 month follow-up to assess their perception of the yoga experience. Participants were between the ages of 30 and 65 years; at baseline, there were no statistically significant differences between groups except for the BDI which was substantially higher in the control group. After 6 weeks, the authors reported that both $46 \%$ of the yoga group and $40 \%$ of the control group were less disabled as measured by the ODI. Regarding depression, however, $54 \%$ of the subjects in the yoga group had lower scores on the BDI compared with only $20 \%$ of the control group. In addition, the SR and FR tests improved in 64 and 90\%, respectively, of the yoga group while only two participants $(20 \%)$ in the control group improved on these measures. Analysis of the qualitative data from the yoga group revealed that group intervention motivated the participants and furthermore, that yoga fostered relaxation and "new awareness." This study was not powered for statistical significance, but there appears to be potentially important trends of decreased depression and improved flexibility and balance in patients with CLBP undergoing a yoga intervention.

In 2005, Williams et al. evaluated the efficacy of an Iyengar yoga intervention compared to an educational control group on pain-related outcomes in patients with CLBP [5]. Forty-four participants with nonspecific LBP of 
3 months duration or longer were randomized to one of the two groups including 20 to the yoga group and 24 to the control group. The yoga intervention was developed by consultation with senior Iyengar instructors who had experience with a yoga protocol for CLBP. Iyengar instructors taught the 90-min class which consisted of a progression of 29 postures weekly for 16 weeks. In addition, yoga participants were encouraged to practice yoga at home for $30 \mathrm{~min}, 5$ days per week. The educational control group received newsletters on back care as well as two 1-h lectures consisting of physical and occupational therapy education.

The primary outcome measure was functional disability, measured with the Pain Disability Index (PDI). Secondary outcome measures included pain intensity assessed with the Short Form-McGill Pain Questionnaire (SF-MPQ); psychological (pain attitudes, fear of movement and selfefficacy) and behavioral (coping strategies) outcomes; spinal range of motion; and pain medication usage. All outcome measures were assessed at baseline, post-treatment and at 3-month follow-up. Finally, adherence to home-based yoga practice was also assessed.

At baseline, there were no significant differences in demographics and medical history between the yoga and the control groups. The average participant had a mean age of 48.3 (range of 23-67 years) and an average duration of LBP for 11.2 years. In addition, there were no significant differences between groups at baseline for most outcome variables. The authors noted that the participants were relatively healthy in terms of pain and disability. Posttreatment, the yoga group had significantly lower PDI scores for functional disability versus baseline compared to the control group with the yoga group's mean scores falling $76.9 \%$ and the control group $39.6 \%$. The greater improvement in functional disability for the yoga group was maintained at a 3-month follow-up. In addition, yoga participants had statistically significant reductions in pain compared to the control group at a 3-month follow-up; the yoga group reported a $70 \%$ decrease in pain compared to a $38 \%$ reduction in the control group. Finally, drug usage post-treatment decreased significantly in the yoga group compared to the control group; $88 \%$ of the participants in the yoga group reported decreasing or stopping their medication compared to $35 \%$ in the control group. At a 3month follow-up, both groups reported further decreases in pain medication usage but the yoga group continued to report significantly greater reductions than the control group. On the other hand, no significant differences were found for the other secondary outcome measures including psychological and behavioral factors and spinal range of motion. Finally, regarding adherence to home practice, participants in the yoga group reported practicing an average of 52 min per week.
The authors concluded that yoga therapy resulted in greater benefits for patients with CLBP compared to an educational program. Specifically, they stated their data showed that a yoga intervention could significantly reduce disability and pain and decrease use of pain medications in such patients. Furthermore, because significant improvements were maintained at 3 months, the authors concluded that yoga was associated with longer lasting reductions in disability and pain than an educational intervention. Regarding the lack of change in psychological and behavioral factors as well as spinal range of motion, the authors stated that their study did not have enough power to obtain statistical significance for these secondary outcomes. In addition, they postulated the duration of time necessary to change long-held negative beliefs such as fear of movement might be longer than the time required to improve perceptions of disability and pain.

The seminal trial of yoga for low back pain

Sherman and colleagues conducted a randomized controlled trial in 2005 comparing the effects of yoga classes to conventional exercise classes and a self-care book in patients with LBP [6]. One hundred and one subjects with nonspecific LBP of at least 12 weeks duration and a history of a recent visit to primary care for this complaint were enrolled in the study. Participants were then randomly assigned to one of the three interventions including 36 participants to the yoga class, 35 to the conventional exercise class, and 30 to the self-care book. Exclusion criteria included subjects with sciatica, spinal stenosis, significant discogenic symptoms, or any ongoing compensation issues. The Viniyoga style of yoga was chosen with each class designed by a senior Viniyoga teacher using a core of 17 postures specifically geared for patients with LBP who did not have any previous yoga experience. For the exercise intervention, a physical therapist created classes incorporating aerobic conditioning, strengthening exercises that emphasized leg, hip, abdominal, and back muscles, and stretching. The instructors taught these 75min classes twice weekly for 12 weeks. In addition, all yoga and exercise class participants were encouraged to practice at home daily. Finally, subjects randomized to the self-care book received an evidence-based book that emphasized self-care strategies for LBP.

Primary outcome measures included back-related functional status assessed with the modified Roland Disability Scale and "bothersomeness" of pain assessed with an 11point numerical scale. Secondary outcome measures included general health status measured with the Short Form-36 Health Survey, degree of restricted activity, and medication usage. All outcome measures were assessed at baseline and 
at 6,12 , and 26 weeks after intervention, with 12 weeks being the primary time point. In addition, safety of the yoga and exercise classes as well as adherence to class and home practice in both of these groups were assessed.

The three groups were generally matched with regard to baseline characteristics. The typical participant was a college-educated, working, white woman between the ages of 40 and 50 years old. Two-thirds of participants reported having back pain for more than 1 year. Few subjects reported work loss or significant activity restrictions due to back pain, but more than one-half had taken medications in the week before the study. The Roland disability score decreased in all three groups over the course of the study; however the mean Roland scores, adjusted for baseline scores, were significantly different among the three groups at all three follow-up time points. The yoga group showed statistically and clinically important improvements in disability at all follow-up points compared to the book group. Although the yoga group had significantly greater improvements in disability compared to the exercise group at 12 weeks, the differences were clinically unimportant. "Bothersomeness" of symptoms also decreased in all three groups during the 12-week intervention period; however, between weeks 12 and 26, symptoms continued to improve only in the yoga group whereas participants in the exercise and book groups experienced worsening symptoms. At 6 and 26 weeks, the yoga group experienced clinically and statistically significant reductions in symptoms compared with the book group. At the primary time point of 12 weeks, however, there were no statistically significant differences among the three groups. Finally, there were no significant differences between the three groups over time for the Short Form-36 Health Survey or degree of restricted activity. Medication usage, however, which was similar among groups at baseline decreased most sharply in the yoga group. Notably, no serious adverse events were reported in the yoga or exercise groups. Finally, class attendance was similar in the yoga (median classes attended, 9) and exercise (8) groups; more than $75 \%$ of participants in both groups also reported practicing at home for an average of three or more days per week.

The authors concluded that yoga was more effective than a self-care book in reducing pain and improving functional status in patients with CLBP. Furthermore, they stated these benefits appeared to persist for at least several months after intervention. On the other hand, while the yoga group consistently reported superior outcomes compared to the exercise group, none of these differences was statistically significant. Finally, the authors noted the yoga intervention was safe and had moderately good adherence. In conclusion, the authors suggested that the Viniyoga style of yoga was an effective and safe treatment for patients with nonspecific CLBP.
Making sense of the yoga literature

Of the four studies discussed above, the Sherman et al. trial is the only study that has high methodological quality and is adequately powered for statistical results. The Galantino and Jacobs studies provide published concept validity for the use of yoga in the management of CLBP. The Williams trial is a small study suggesting statistical trends for the efficacy of yoga above an educational group in individuals with nonspecific LBP. Overall, the available literature suggests that a yoga intervention may decrease pain and improve function in the CLBP population. In addition, yoga appears to be a safe treatment option for the LBP patient with moderately good adherence.

The precise mechanism that underlies the therapeutic effects of yoga is unclear, but it appears to work on physical and mental factors that are associated with LBP. Yoga is generally believed to improve flexibility, strength, balance, and agility. For patients with LBP, yoga appears to address imbalances in the musculoskeletal system affecting spinal alignment and posture. For example, yoga targets many muscle groups with the aim of lengthening tightened muscles and strengthening often underutilized core muscles. In fact, in studies of patients with LBP, yoga has been found to increase hip flexion as well as spinal and hamstring flexibility $[4,7]$. As discussed above, however, the practice of yoga places as much emphasis on relaxation and meditation as on physical movement. The mental focus induced by yoga likely helps people increase their awareness of how they position and move their body in maladaptive ways and relax tense muscles. In addition, yoga is generally believed to reduce stress and improve mood and overall well-being; these effects are likely enhanced by the breathing techniques taught as part of the yoga practice.

\section{Pilates}

Pilates is another popular form of mind-body exercise where the focus is on controlled movement, posture, and breathing. Joseph H. Pilates developed the comprehensive program known as the Pilates method in the 1920's. The dance community initially embraced Pilates as a method of conditioning ballet and modern dancers. Over the last decade, the popularity of this exercise has grown, and Pilates is now used as both a form of fitness and holistic health. There are no published statistics regarding the number of people who practice Pilates in the United States today; however like yoga, Pilates classes now have a ubiquitous presence in health and fitness clubs. In addition, private Pilates studios are common and typically offer both group mat classes as well as private sessions on specialized apparatus. 


\section{Pilates background}

Similar to yoga, the Pilates method incorporates both physical and mental elements. The technique focuses on the "power house" or what is known today as the core; in Pilates, this includes the abdominal, gluteal, and paraspinal muscles in particular. Pilates exercises involve progressive multiplanar excursion of the trunk and limbs. Each starts by stabilizing the core musculature and then proceeds through a controlled range of motion (see Figs. 3 and 4). The goals are to increase muscle strength and endurance as well as flexibility and to improve posture and balance. The mental element of Pilates is evident in the additional focus on breathing and concentration during the execution of these exercises. Exercises are performed both on the mat as well as on specialized equipment. In the mat class, participants typically sit or lie supine or prone and use gravity to help stabilize the core. Of note, many of these exercises are nonweightbearing and have a strong flexion bias. In addition, Mr. Pilates designed specialized apparatus to train a variety of movement patterns and postures. The Reformer, for example is made of a sliding horizontal platform within a box-like frame upon which a person sits, stands, kneels or reclines; varying resistance to movement is provided via light springs attached to the moving platform and through a simple pulley system. Other commonly used pieces of Pilates equipment include the Barrel, Cadillac, and Wunda Chair.

\section{The Pilates literature}

The Pilates method has been increasingly applied for its therapeutic benefits, however little scientific evidence supports or rebukes its use as a treatment regimen for musculoskeletal diagnoses including LBP. While most evidence to date is testimonial or in the form of uncontrolled case series, two randomized controlled studies exist regarding the effect of Pilates on patients with nonspecific

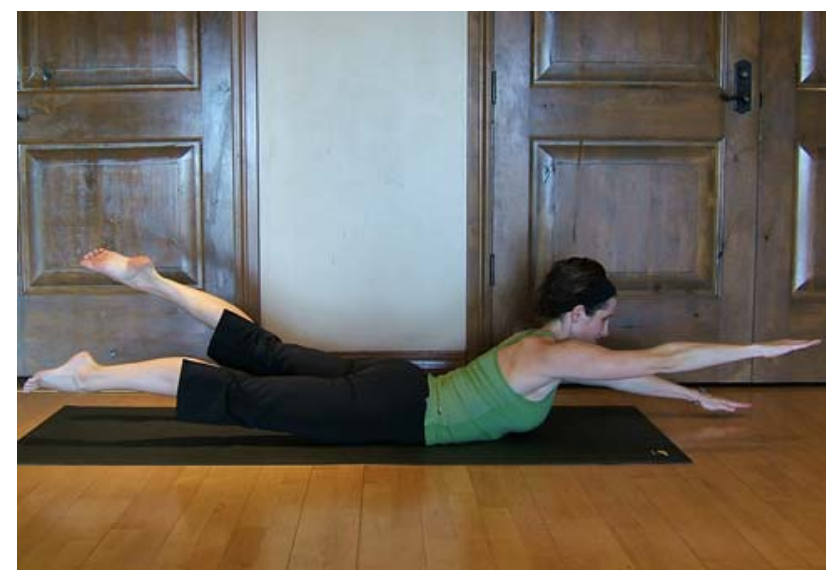

Fig. 3 Pilates exercise-Swimming

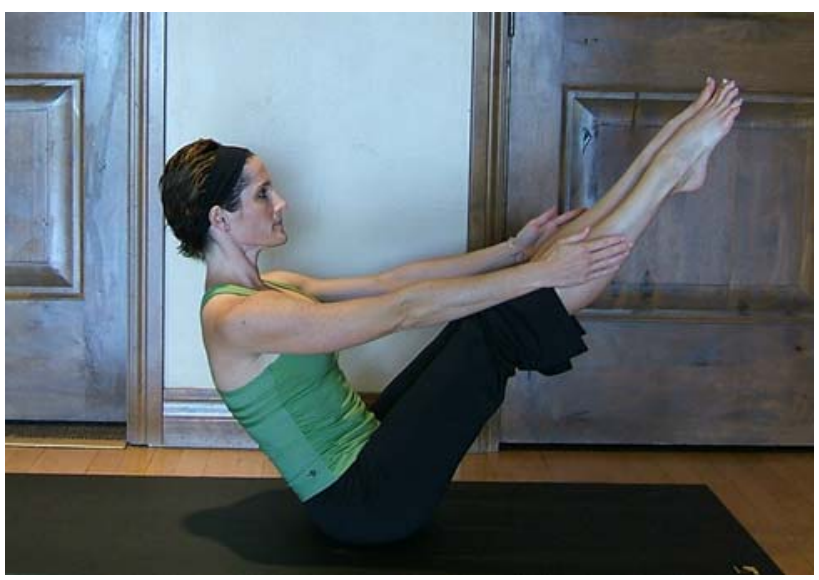

Fig. 4 Pilates exercise-Teaser

CLBP. Donzelli et al. published a randomized controlled study in 2006 to evaluate the efficacy of a Pilates method called Pilates CovaTech compared to a Back School intervention for patients with LBP [8]. The CovaTech method is a specific rehabilitation method utilized in Italy derived from the original Pilates method. Forty-three subjects who had nonspecific LBP for at least 3 months and were receiving treatment for this complaint were enrolled in this study. Participants were then randomly placed into one of the two groups including 21 to the Pilates CovaTech method and 22 to the Back School method. A rehabilitation therapist trained in the particular method taught a one-hour class to a group of up to seven subjects for 10 consecutive days. For the Pilates CovaTech method, the authors stated their protocol comprised several exercise modules including "postural education, search for neutral position, sitting exercises, antalgic exercises, stretching exercises, proprioceptivity improvement exercises, breathing education, and mobilization of the cervical rachis and the scapulahumeral joint." On the other hand, their Back School protocol included "postural education exercises, respiratory education, muscular extension and strengthening exercises of the paravertebral muscles and lower limb, mobilizing exercises for the spinal column, and antalgic postures." Participants in both groups were also encouraged to practice their respective exercises at home.

Outcome measures included disability assessed using the Oswestry Low Back Pain Disability Questionnaire (OLBPDQ) and pain evaluated with the VAS. Assessments were made at baseline and then at 1, 3, and 6-month follow-ups. Subjective response to treatment (worse to better), adherence to home practice, level of satisfaction (dissatisfied to very satisfied), and benefit perceived (little benefit to great benefit) were also assessed in both groups, however no statistical analysis was applied to these results.

At baseline, demographic and clinical characteristics were similar between groups with a mean age of 50 years 
(range of 20-65 years). Regarding outcome measures, there were significant reductions in both disability and pain intensity in both groups. In the Pilates group, average OLBPDQ scores showed a sharper but nonsignificant fall in the first month versus baseline values compared to the Back School group. The mean improvement, however, between baseline values and those obtained at the final 6month visit was similar in both groups. In addition, the participants in the Pilates method reported a better subjective response to treatment as compared to those in the Back School method at all follow-up time points. For adherence to home practice, only $26 \%$ of the total participants performed any home-based exercises with just $7 \%$ performing them on a regular basis. Regarding level of satisfaction, $62 \%$ of participants in the Pilates Method stated they were "very satisfied" compared to $4.5 \%$ of those in the Back School; for benefit perceived, participants in both groups showed similar results.

The authors concluded that the equally good results obtained with the Pilates CovaTech method demonstrated it was as efficacious as the Back School method in terms of both short- and long-term (6 month) outcomes. They also stated the Pilates intervention had better compliance because of subjective responses such as improvement of symptoms and satisfaction with treatment. In conclusion, the authors proposed the Pilates CovaTech method was a valid alternative in the management of patients with nonspecific CLBP.

Lastly, Rydeard et al. published a randomized controlled study in 2006 on the effects of a Pilates-based therapeutic exercise protocol on patients with CLBP as compared to a control group receiving usual care [9]. Thirty-nine subjects with nonspecific CLBP were enrolled in this study. Participants were required to have had persistent LBP for greater than 6 weeks or recurring LBP with at least two painful episodes per year "of sufficient intensity to restrict functional activity." They were also required to be physically active, defined by the authors as participating in a minimum of three moderately intense $30 \mathrm{~min}$ sessions of activity per week. Finally, subjects had to exhibit strength of $4 / 5$ or less on manual muscle testing of the gluteus maximus as well as altered recruitment of this muscle as determined by visual and manual inspection during a prone leg extension test. These individuals were then randomized to one of the two groups including 21 to the Pilates group and 18 to the control group. The Pilates protocol consisted of exercises performed on the mat and the Reformer that were designed to train the activation of specific muscles thought to stabilize the lumbar-pelvic region. Subjects recruited the pelvic floor and lumbar multifidus and then activated the gluteus maximus during a variety of movement patterns involving hip extension. Movements were initially performed in supine with the lumbar spine in neutral and then progressed to more upright postures with movement out of neutral postures. One of the two physical therapists trained in this protocol taught the $1 \mathrm{~h}$ session 3 times weekly for 4 weeks. In addition, the Pilates participants were asked to complete a 15-min home program 6 days per week which involved similar exercises performed on the floor. The control group received usual care as defined by the authors as "consultation with physicians and other health care professionals as necessary." The control group was also instructed to continue participating in their usual physical activity.

Outcome measures included functional disability assessed with the 24-point Roland Morris Disability Questionnaire (RMQ) and average pain intensity measured with a 101-point numerical rating scale. Outcomes were evaluated at baseline and end of treatment in both groups; in addition, retention of treatment effect for disability was tested at 3,6, and 12 months post-treatment in the Pilates group. Both groups were matched regarding baseline characteristics. In the entire group, subjects were aged 2055 years and had a median duration of symptoms of 8.2 years; $20 \%$ reported recurring LBP while $80 \%$ reported persistent LBP. Of note, $90 \%$ of all participants reported having received previous physical therapy intervention and $74 \%$ of those interventions had included exercise-based therapy. There were significantly lower levels of disability and pain following intervention in the Pilates group compared to the control group. For functional disability, the mean RMQ score in the Pilates group was 2.0 compared to 3.2 in the control group at end of treatment. For average pain intensity, the mean number in the Pilates group was 18.3 compared to 33.9 in the control group at end of treatment. In addition, improved disability scores were maintained in the Pilates group for up to 12 months post-treatment.

The authors concluded that treatment with a modified Pilates-based approach was more efficacious in reducing functional disability and pain intensity than usual care in a population with CLBP. They noted a major limitation of their study was the results were potentially not generalizable to other CLBP patients who had more disabling pain precluding them from being as physically active or did not have altered performance of the gluteus maximus. On the other hand, they stated their particular group of patients likely represented individuals who sought ongoing treatment from healthcare providers for CLBP after they returned to normal daily activities with still some restrictions and limitations.

Making sense of the Pilates literature

Since the literature review on the subject of Pilates and LBP yielded only two randomized controlled studies, it is 
difficult to reach a firm conclusion regarding the efficacy of this exercise intervention for such patients. These two studies, however, do suggest that Pilates has beneficial effects in terms of decreasing pain and disability in patients with nonspecific CLBP. Similar to yoga, the exact mechanism by which Pilates exerts these benefits has not been elucidated. The Pilates method does, however, utilize principles of various accepted rehabilitation methods that have scientific support for LBP including core strengthening [10, 11]. Core weakness has been increasingly recognized as a biomechanical deficit in patients with LBP; however, muscle dysfunction here may not simply be a problem of muscle strength. Instead, the problem appears to involve poor neuromuscular control, or recruitment patterns of core musculature, which negatively affects spinal stability. Therefore, specifically designed therapeutic exercise approaches that modulate neuromuscular control and enhance spinal stability have been investigated for patients with LBP. For example, O'Sullivan et al. evaluated the efficacy of a rehabilitation technique that enhances lumbar spine stabilization via muscle activation directed at the intervertebral segment in patients with CLBP who also had signs of radiological instability [10]. Significant reductions in pain and functional disability were shown in these patients; these effects were maintained at 30-month follow-up. It follows the Pilates method would be beneficial for patients with LBP because it improves absolute core strength and moreover encourages proper activation patterns of core musculature. For example, the foundation of the mat program is a group of exercises that train core stabilization; when proper recruitment patterns are demonstrated, more challenging exercises are progressively added to improve core strength. In addition, the modified Pilates approach developed by Rydeard et al. with its emphasis on specific muscle activation strategies to stabilize the lumbar-pelvic region is supported within this theoretical context of neuromuscular control and spinal stability. The effects of this approach on decreasing pain and improving function in patients with CLBP who also have dysfunction in control of muscles stabilizing the lumbar-pelvic region are in agreement with the O'Sullivan findings. Finally, in addition to the role core strengthening plays in benefiting patients with CLBP, one must also consider the impact of the mental component of Pilates. Like yoga, the Pilates' principles of breathing and concentration are no doubt intricately tied to the mechanism behind its effectiveness for the CLBP population.

\section{Conclusion}

In conclusion, the studies outlined in this chapter are an important start in the evaluation of how yoga and Pilates may be helpful in managing patients with LBP. It is important to note, however, that all of the above trials focus on nonspecific LBP. Future studies should therefore examine the efficacy of postures and exercises geared specifically for different LBP diagnoses as there may be varying clinical effect. For example, an individual with flexion mediated low back and leg pain secondary to lumbar radiculopathy from a herniated disc may benefit more from movements that emphasize extension versus those that involve flexion and/or twisting. In addition, trials should incorporate larger sample sizes to obtain more power for statistical significance. Different clinical arms should be employed comparing for example, yoga or Pilates to physical therapy. Control groups should include group and individualized formats to detect any benefit that may be derived from group support including socialization context. Longer follow-up should be assessed for outcome measures to determine the long-term effects of these exercise interventions. Finally, studies should attempt to elucidate the mechanisms that underlie the benefits of these exercise interventions for patients with LBP.

Given the interest of the general population in yoga and Pilates and the emergence of information supporting their use for CLBP, it appears these mind-body exercise therapies may be helpful tools in managing patients with LBP. Health care providers who treat LBP and consider recommending yoga and/or Pilates to these patients should therefore have a basic understanding of these exercise regimens. For yoga, one should be familiar with the core postures of a typical yoga class. One should also understand that different styles of yoga exist and some, i.e. Iyengar yoga and Viniyoga, may be better suited for the LBP patient. For Pilates, one should be aware of the basic exercises in a mat class as well as the potential for progression to more challenging exercises on specialized apparatus. Finally, because instructors are not required to obtain a specific license in order to teach yoga or Pilates, one should direct the patient to seek out instructors who have at least several years of teaching experience including working with LBP patients. With this information, the health care provider will be well equipped when recommending yoga or Pilates as a management tool for this often challenging group of patients.

\section{References}

1. Hayden JA, van Tulder MW, Malmivaara A, Koes BW. Exercise therapy for treatment of nonspecific low back pain. Cochrane Database Syst Rev. 2000; CD000335.

2. Saper RB, Eiseberg DM, Davis RB, Culpepper L, Phillips RS. Prevalence and patterns of adult yoga use in the United States: results of a national survey. Altern Ther Health Med 2004;10:449. 
3. Jacobs BP, Mehling W, Goldberg HA, Eppel E, Acree M, Lasater $\mathrm{JH}$ et al. Feasibility of conducting a clinical trial on Hatha yoga for chronic low back pain: methodological lessons. Altern Ther Health Med 2004;10:80-3.

4. Galantino ML, Bzdewka TM, Eissler-Russo JL, Holbrook ML, Mogck EP, Geigle P et al. The impact of modified Hatha yoga on chronic low back pain: a pilot study. Altern Ther Health Med 2004;10:56-9.

5. Williams K, Steinberg L, Petronis J. Therapeutic application of Iyengar yoga for healing chronic low back pain. Int J Yoga Ther 2003;13:55-67.

6. Sherman KJ, Cherkin DC, Erro J, Miglioretti DL, Deyo RA. Comparing yoga, exercise, and a self-care book for chronic low back pain: a randomized, controlled trial. Ann Intern Med 2005;143:849-56.

7. Williams KA, Petronis J, Smith D, Goodrich D, Wu J, Ravi N et al. Effect of Iyengar yoga therapy for chronic low back pain. Pain 2005;115:107-17.
8. Donzelli S, Di Domenica F, Cova AM, Galletti R, Guinta N. Two different techniques in the rehabilitation treatment of low back pain: a randomized contolled trial. Eura Medicophys 2006;42:205-10.

9. Rydeard R, Leger A, Smith D. Pilates-based therapeutic exercise: effect on subjects with nonspecific chronic low back pain and functional disabililty: a randomized controlled trial. J Orthop Sports Phys Ther 2006;36:472-84.

10. O'Sullivan PB, Phyty GD, Twomey LT, Allison GT. Evaluation of specific stabilizing exercise in the treatment of chronic low back pain with radiologic diagnosis of spondylolysis or spondylolisthesis. Spine 1997;22:2959-67.

11. Akuthota V, Nadler SF. Core strengthening-review. Arch Phys Med Rehabil 2004;85:S86-92. 